\title{
Study of role of digital breast tomosynthesis over digital mammography in the assessment of BIRADS 3 breast lesions
}

Engy A. Ali ${ }^{1,2^{*}}$ and Lamiaa Adel $^{2}$

\begin{abstract}
Background: Breast cancer is the most common malignancy in women and thus, screening has become an important health issue. Although mammography remains the standard of care for breast cancer screening and diagnosis (with biopsy), tomosynthesis (3D DBT) allows the separation of overlapping structures seen on 2D mammography and thus enables better depiction of masses or asymmetries.
\end{abstract}

Results: A prospective study for mammographic cases referred to our radiology unit included 60 lesions detected in 59 patients that were performed during the period from January 2016 to September 2017. Patients' ages ranged from 26 to 72 years with mean age $51 \pm 12$ SD. Sixty percent of breast imaging-reporting and data system (BIRADS) 3 lesions detected by 2D digital mammography (36/60) changed their category after 3D DBT, 40\% (24/60) digital mammography noticed lesions did not change their BIRADS after 3D DBT. Twenty-nine BIRADS 3 lesions out of the 60 were downgraded to BIRADS 1and 2, while 7 BIRADS 3 lesions out of the 60 were upgraded to BIRADS 4 and 5 which were all biopsied. Six out of the 7 lesions were pathologically proven ducal carcinoma and 1 out of 7 pathologically proven to be atypical ductal hyperplasia.

Conclusion: 3D DBT significantly reduced the need for additional mammographic views and frequent follow-up studies as it gave better characterization for all BIRADS 3 lesions.

Keywords: Breast cancer, Digital mammography, Digital breast tomosynthesis, BIRADS

\section{Background}

Breast cancer is the most common cancer in ladies throughout the world, with nearly about 1.7 million new cases detected in 2012 representing about $12 \%$ of all new cancer cases and $25 \%$ of all cancers in female patients [1].

Accurate diagnosis is a must for efficient treatment and for better prognosis. Mammography is still the first breast imaging investigation despite its well-known limitations. However, the known lack of sensitivity is due to the misinterpretation of both architectural distortion and asymmetrical

\footnotetext{
* Correspondence: drengyali78@gmail.com

${ }^{1}$ Radiology Department (Women's Imaging Unit), Kasr El-Ainy Hospital, Cairo University, Giza, Egypt

2Diagnostic and Intervention Radiology Department, Cairo University

Hospitals, Kasr Al-Ainy El-Manial, Cairo 11956, Egypt
}

density as well as the cancer overlapped by fibro-glandular tissue resulting in hiding the cancer margins [2].

Despite technical advances, the sensitivity of mammography is significantly reduced by high breast density. So in patients with breast density c or d, mammographic sensitivity is just $30 \%$ and the interval cancer odds ratio (OR) was 6.14 [3].

Digital breast tomosynthesis (DBT) is a new imaging modality for improving the detection of breast cancer as it provided better detection of the abnormalities especially in females with dense breast and diagnosis of benign lesions resulted in reduction of the recalled cases and negative biopsies as well as assessing efficacy of therapy [4], as well as it enabled visualization of cancers not visualized by conventional mammography [5]. 
Table 1 BIRADS assessment categories according to BIRADS atlas 2013

\begin{tabular}{ll}
\hline Category & Assessment \\
\hline BIRADS 0 & $\begin{array}{l}\text { Incomplete-need additional } \\
\text { imaging evaluation and/or prior } \\
\text { mammograms for comparison }\end{array}$ \\
BIRADS 1 & Negative \\
BIRADS 2 & Benign \\
BIRADS 3 & Probably benign \\
BIRADS 4 & Suspicious \\
& 4A: low suspicion for malignancy \\
& 4B: moderate suspicion for \\
& malignancy \\
BIRADS 5 & 4C: high suspicion for malignancy \\
BIRADS 6 & Highly suggestive of malignancy \\
\hline
\end{tabular}

Digital breast tomosynthesis (DBT) has been heralded as an advancement of mammography by virtue of its significant improvements on performance outcomes. It is distinct from supplemental screening tools such as ultrasound and magnetic resonance imaging (MRI) as its use significantly increases cancer detection while simultaneously reducing recall rates. Single-site and multi-site studies have validated DBT's potential to benefit all women, regardless of age or breast density. In addition, long-term studies are beginning to reflect that the

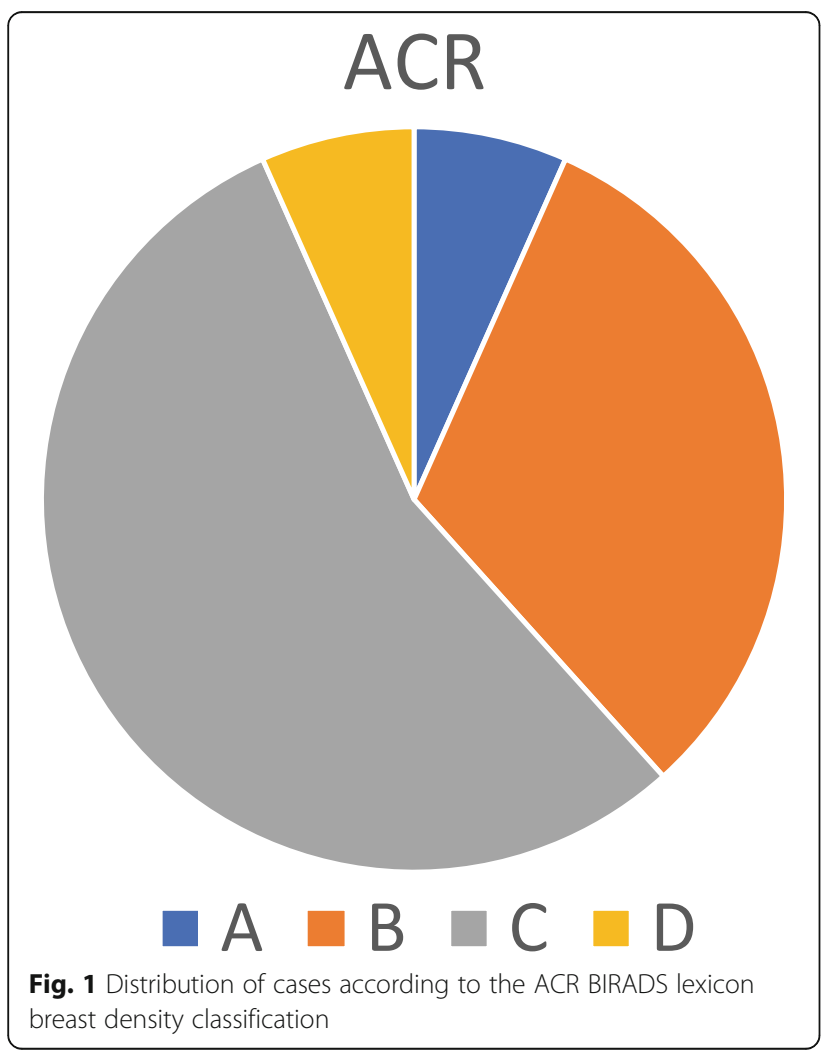

Table 2 Age distribution of the patients participating in the study

\begin{tabular}{lc}
\hline Age & Years \\
\hline Minimum & 26 \\
Maximum & 72 \\
Mean & 51 \\
SD & \pm 12 \\
\hline
\end{tabular}

improvements seen with DBT are not bound to prevalent screening but persist on incident screening. All this has made DBT increasingly requisite at many institutions, both community and academic [6].

Digital breast tomosynthesis (DBT) has improved conventional mammography by increasing cancer detection while reducing recall rates. However, these benefits come at the cost of increased radiation dose. Synthesized mammography (s2D) has been developed to provide the advantages of DBT with nearly half the radiation dose. Since its F.D.A. approval, multiple studies have evaluated the clinical performance of s2D. In clinical practice, s2D images are not identical to conventional 2D images and are designed for interpretation with DBT as a complement [6].

The 2011 F.D.A. approval of DBT specified its performance in conjunction with 2D full-field digital mammography (FFDM): a 2D+DBT combined exam. While still below federal radiation safety limits of $3 \mathrm{mGy}$ per view, a two-view combined exam is, however, approximately double the dose of the 2D FFDM exam alone. Furthermore, this precludes women with implants or large breasts requiring tiling, from receiving DBT, as their screening exams already include additional views. In addition, though the patient may remain in the same compression, the DBT and conventional 2D exams are acquired sequentially, with the addition of DBT adding $3.7-25 \mathrm{~s}$ onto the conventional $2 \mathrm{D}$ exam, depending on vendor [6].

Synthesized mammography (s2D) was created in response to these challenges, with the goal of affording patients the benefits of tomosynthesis, minus the additional radiation and acquisition time, and which first gained US Food and Drug Administration (FDA) approval in 2013. To evaluate $\mathrm{s} 2 \mathrm{D}+\mathrm{DBT}$ as a valid clinical tool, the synthesized

Table 3 Distribution of cases according to the ACR BIRADS lexicon breast density classification

\begin{tabular}{lll}
\hline ACR breast density & Number of lesions & Percentage \\
\hline A & 4 & 6.78 \\
B & 19 & 32.20 \\
C & 33 & 55.93 \\
D & 4 & 6.78 \\
Total & 60 & 100 \\
\hline
\end{tabular}




\section{Lesion character}

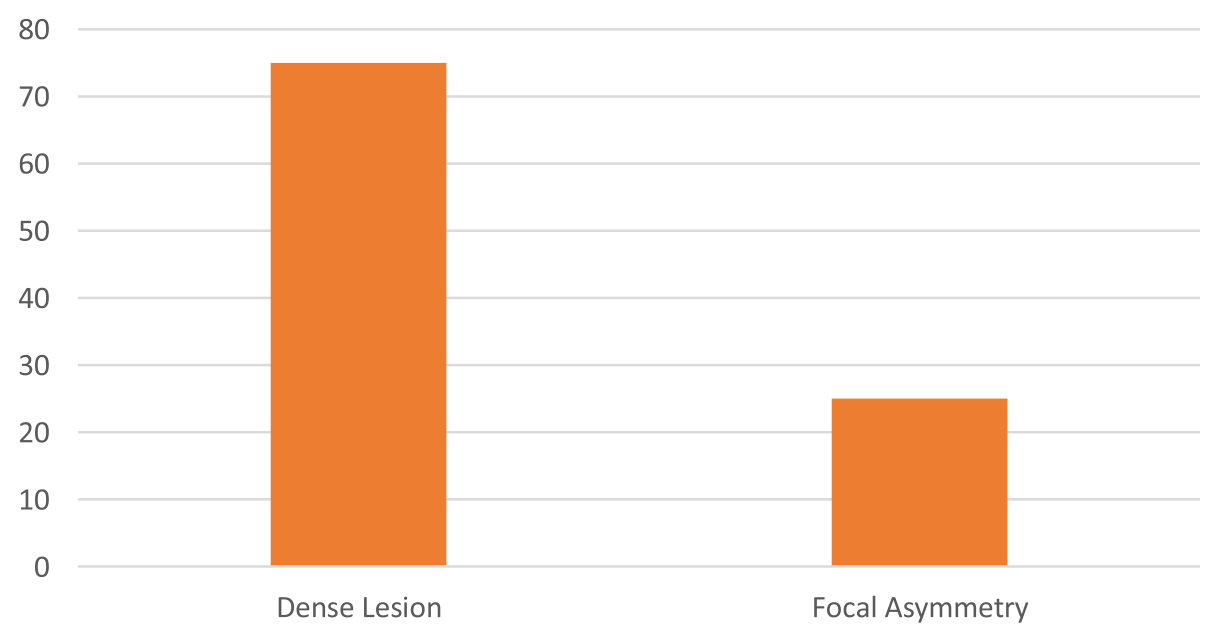

Fig. 2 Lesion character

mammography literature is reviewed here and appraised in accordance with known screening criteria. Implementation suggestions and case examples are also provided herein to ease transition for radiologists considering s2D+DBT as a replacement for 2D+DBT [6].

\section{Aim of the work}

The purpose of this study is to assess the role of 3dimensional breast tomosynthesis in diagnosis and consequently management of breast imaging-reporting and data system (BIRADS) 3 breast lesions.

\section{Methods}

\section{Patients}

A prospective study, done in New kasr El Aini Teaching Hospital including 59 cases, all underwent digital mammography with 2D and 3D mammography acquisitions, with the use of software that allowed synthetic $2 \mathrm{~d}$ mammographic images to be reconstructed from 3D acquisitions.

Mammography reading was done in two parallel double reading conducted sequentially for 2D acquisitions followed by integrated acquisitions.

The study was performed in 59 cases, detecting 60 BIRADS 3 lesions. All mammograms were classified as BIRADS1-5 category after 3D DBT (Table 1). A complementary ultrasound examination was performed for all cases to confirm or exclude mammography identified abnormalities using high frequency probe. All breast lesions that were upgraded by 3D DBT were either aspirated, biopsied, or surgically removed, and followed-up.

All patients were subjected to demographic and clinical data which includes patient's name, age, marital status and number of offsprings, lactating history, residence and phone number, diagnosis, past history, and family history.

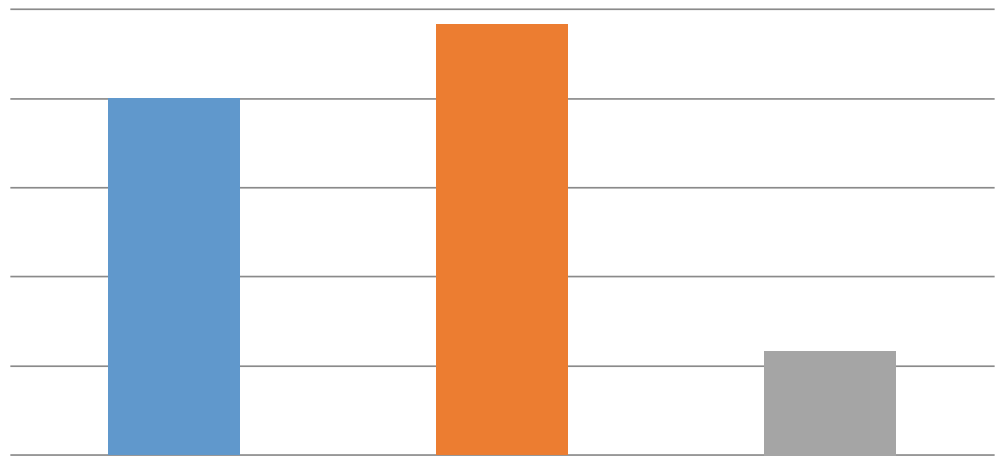

Fig. 3 Tomosynthesis outcome on the BIRADS classification 
Table 4 BIRADS 3 lesions following DBT

\begin{tabular}{llllllll}
\hline DM & \multicolumn{3}{l}{ BIRADS DBT } & & & & No. \\
BIRADS 3 & 1 & 2 & 3 & 4 & 5 & \\
\hline & 2 & 27 & 24 & 7 & 0 & 60 \\
& & & & & & &
\end{tabular}

\section{Inclusion criteria}

Women presenting with abnormal findings by conventional mammography and assigned BIRADS 3 lesions

\section{Equipments}

Mammographic examination was performed using Senographe Essential, GE healthcare full-field digital mammography machine with 3D digital breast tomosynthesis, with the use if software that allowed synthetic 2D mammographic images to be reconstructed from 3D acquisitions.

\section{Technique of full field digital mammography}

Standard views medio-lateral-oblique and cranio-caudal views were taken for all patients.

\section{Technique of 3D tomosynthesis}

For 3D digital tomosynthesis, two views (MLO and CC) were obtained. Three-dimensional DBT involved the acquisition of 12 to $152 \mathrm{D}$ projection exposures by a digital detector from a mammographic x-ray source which moves over a limited arc angle, with the use of software that allowed synthetic $2 \mathrm{~d}$ mammographic images to be reconstructed from 3D acquisitions.

Mammography reading was done in two parallel double reading conducted sequentially for $2 \mathrm{D}$ acquisitions followed by integrated acquisitions.

\section{Image analysis and interpretation of mammography and 3D digital tomosynthesis}

1. Breast density was assessed for each patient.

Table 5 BIRADS 3 lesions following DBT and breast cancer cases

\begin{tabular}{llll}
\hline DBT & DM & Breast cancer & \\
\cline { 3 - 4 } BIRADS & BIRADS & Yes & No \\
& 3 & 0 & 2 \\
\hline BIRADS 1 & 2 & 0 & 27 \\
BIRADS 2 & 27 & 0 & 24 \\
BIRADS 3 & 24 & $6(85.71 \%)$ & 1 \\
BIRADS 4 & 7 & 0 & 0 \\
BIRADS 5 & 0 & 6 & 54 \\
Total & 60 & & \\
\hline
\end{tabular}

2. Each lesion was evaluated regarding site and type (mass, focal asymmetry \pm calcifications and size).

3. Lesions were classified as benign or malignant according to the mammography BIRADS lexicon morphology descriptors:

(a) Mass lesions: shape, margin, density, and size

(b) Asymmetry: simple, focal, global, or developing

(c) Calcifications: morphology and distribution.

4. Two readers independently read synthetic $2 \mathrm{DM}$ and 3DBT. As a part of the diagnostic procedure, we determined the BIRADS category of the lesions in each of the imaging modalities individually according to the BIRADS lexicon 2013 classification (Fig. 1).

\section{Results}

A prospective study for mammographic cases referred to our radiology unit included 60 lesions detected in 59 patients that were performed during the period from January 2016 to September 2017.

\section{Age}

Patients' ages ranged from 26 to 72 years with mean age $51 \pm 12$ SD (Table 2).

\section{Breast density ACR scoring}

According to the 2013 American College of Radiology BIRADS lexicon classification of breast density ACR "a" indicates that the breasts are almost entirely fatty, ACR " $b$ " indicates that there are scattered areas of fibroglandular density, ACR " $\mathrm{C}$ " indicates that the breasts are heterogeneously dense, and ACR " $d$ " indicates that the breast is extremely dense (Table 3 ).

According to the ACR BIRADS lexicon breast density classification:

Table 6 BIRADS classification by DBT percentage change

\begin{tabular}{lll}
\hline BIRADS & DBT & \\
\cline { 2 - 3 } & No. & Percent \\
\hline 1 & 2 & $3.33 \%$ \\
2 & 27 & $45 \%$ \\
3 & 24 & $40 \%$ \\
4 & 7 & $11.66 \%$ \\
5 & 0 & 0 \\
Total & 60 & 100 \\
\hline
\end{tabular}


- 4/60 (6.78\%) lesions were assigned an ACR score of "a".

- 19/60 (32.20\%) lesions were assigned an ACR score of "b".

- 33/60 (55.93\%) lesions were assigned an ACR score of "c".

- 4/60 (6.78\%) were assigned an ACR score of "d".

\section{Lesion character}

Seventy-five percent of the cases had a dense lesion and $25 \%$ had focal asymmetry by digital mammography (Fig. 2).

\section{BIRADS classification}

Sixty percent of BIRADS 3 lesions detected by 2D digital mammography (36/60) changed their category after 3D DBT and 40\% (24/60) of digital mammography noticed lesions did not change after 3D DBT. Sixty percent of the lesions changed their BIRADS system classification following DBT (Fig. 3):

1. 3D DBT down staged to BIRADS 1 and 2 in 29 lesions representing $48.33 \%$ of the cases.

2. 3D DBT upstaged to BIRADS 4 (or more) in 7 lesions representing $11.7 \%$ of the cases.
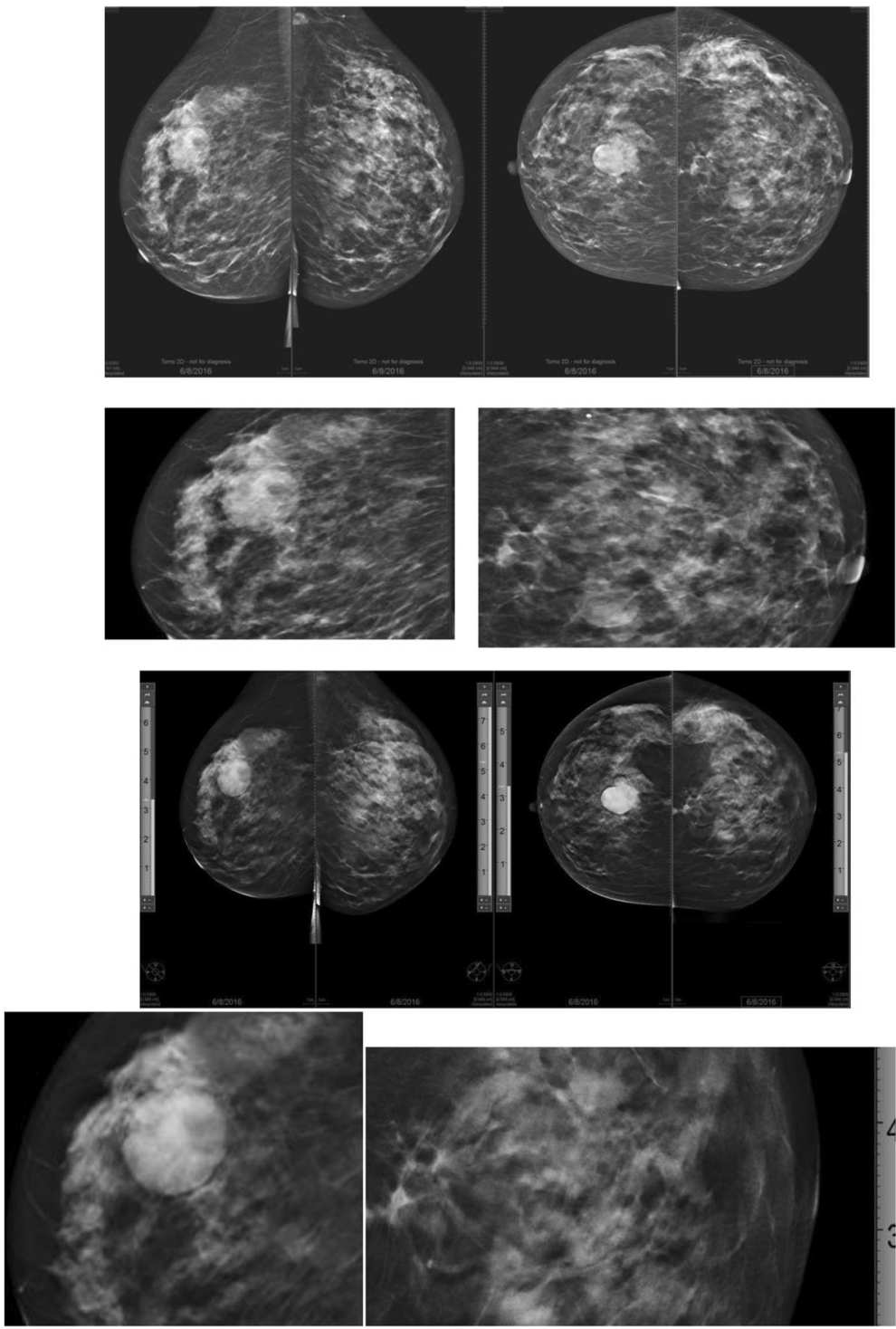

Fig. 4 Sixty-five-year-old female mammography CC and MLO views ACR C .Bilateral central partially well-circumscribed partially obscured dense lesions, BIRADS 3. Lt upper central and UOQ area of focal asymmetry and architectural distortion, BIRADS 3. Tomosynthesis CC and MLO views right central rather well-circumscribed dense lesion, BIRADS 2. Left upper central irregular speculated lesion BIRADS 4C. 3D digital breast tomosynthesis easily detected the lesion and this changed the BIRADS from 3 to 2, proved by US to be cyst, tomosynthesis upgraded the left central lesion which was proved to be duct carcinoma by biopsy 
Forty percent of the lesions did not change their BIRADS classification after 3D DBT.

We performed a biopsy in all suspected findings on tomosynthesis. 3D DBT detected 6 cancers in 7 lesions BIRADS 4 category, that were proved to be ductal carcinoma by biopsy, and one lesion was proved to be atypical ductal hyperplasia.

The following tables show the BIRADS grade for breast lesions by the technique used (mammography, tomosynthesis) (Tables 4, 5, and 6).

\section{Case presentation}

\section{Discussion}

Mammography was still the best screening imaging modality for the women worldwide. The decision of starting screening should be the result of personalized discussion between the female patient and her provider, including both the benefits and risks of routine screening [7].

$3 D$ digital breast tomosynthesis (3D DBT) was a new screening and diagnostic technique in breast evaluations with a high promising role in increasing the sensitivity and specificity of digital mammography (DM) [8].

Women with dense breast were usually at high risk of developing breast cancer as the increase density might mask the radiological signs of cancer, while on the other hand cancers were clearly visible in fatty breasts on mammogram. So the detection of breast carcinoma might be complex and in need of multiple imaging modalities [2].
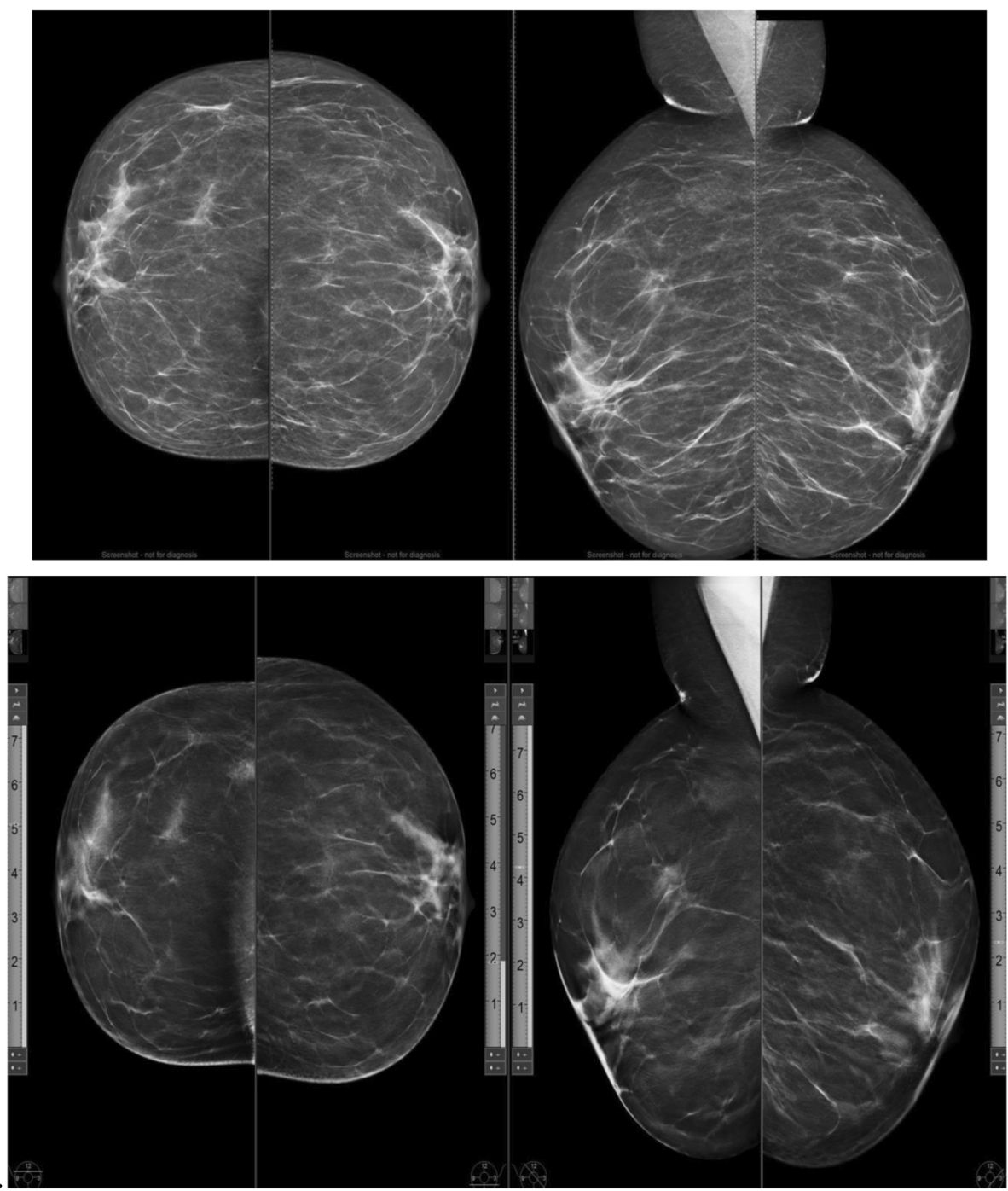

Fig. 5 Fifty-three-year-old female. Mammography CC and MLO view. Scattered fibro-glandular breasts parenchyma (ACR B). Focal asymmetry is noted at the UOQ of the right breast BIRADS 3. No dominant spiculated masses or pathological micro calcifications seen. Tomosynthesis of both breasts CC and MLO views. The upper outer area of focal asymmetry was confirmed to be an area of over lapping fibro-glandular tissue with dirty lucencies appearance, BIRADS 2. Tomosynthesis downgraded the BIRADS from 3 to 2 
3D DBT was one technology being developed to improve both the detection and characterization of the breast lesions mainly in females with non-fatty breasts as it was expected to overcome the limitations of mammography caused by overlapping of both normal and pathological tissues during the standard two-dimensional (2D) imaging projections [7].

The known benefits of 3D DBT improved in both screening sensitivity and detection of lesion size as well
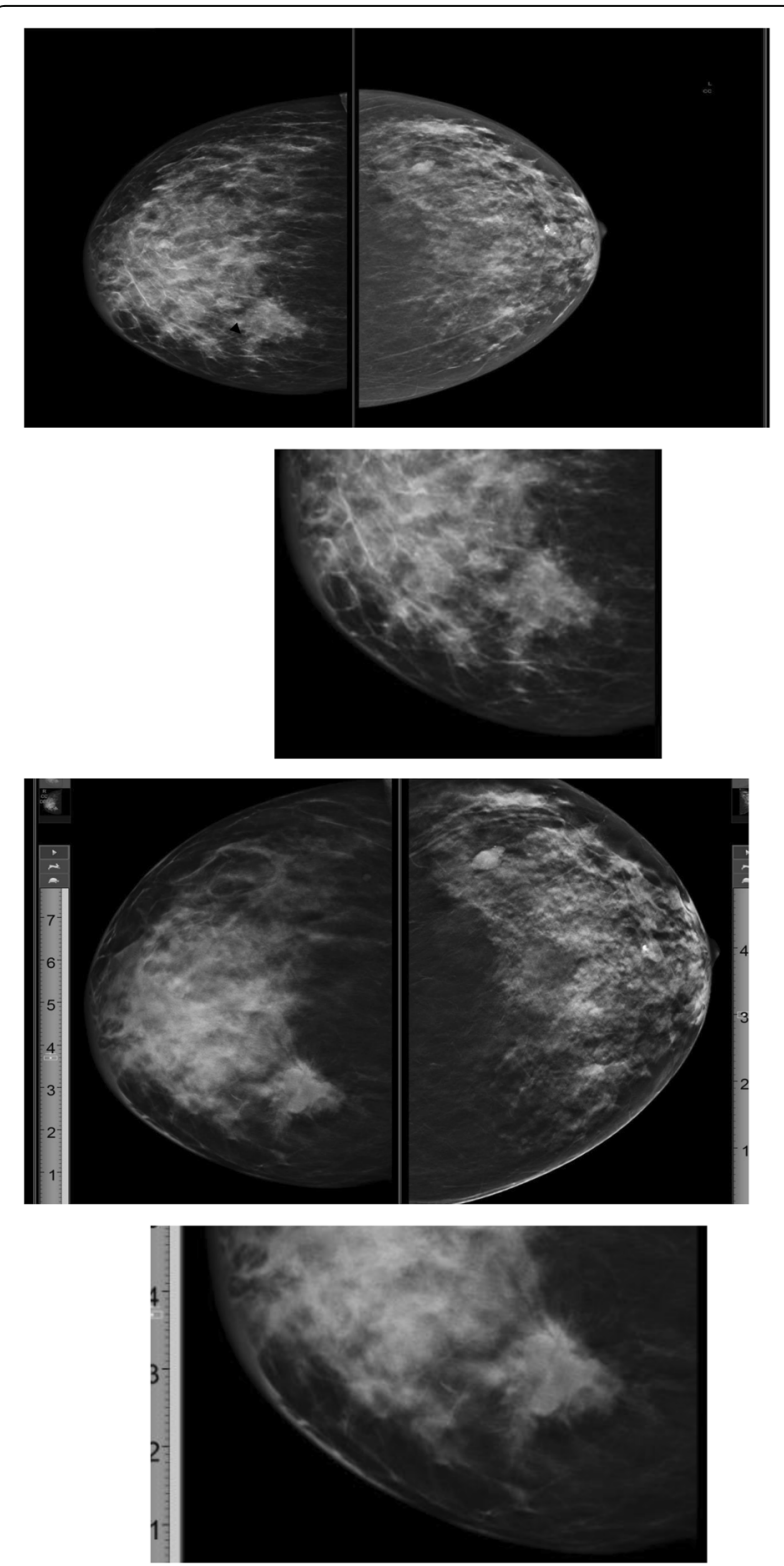

Fig. 6 Sixty-one-year-old female. Mammography CC view ACR C, RT UIQ obscured dense lesion, BIRADS 3. Tomosynthesis CC view Rt UIQ irregular speculated dense lesion, BIRADS 4C. The margin of the lesion appeared speculated on 3D digital tomosynthesis images (BIRADS 4), which was proved to be duct carcinoma as lesion characterization, which in turn resulted in decreasing the recall rates [5].

Our aim of study was to detect and prove the role of 3D digital tomosynthesis in evaluation of BIRADS 3 breast lesions.

Bunovic et al. performed a prospective study in which 3D DBT was performed in 360 DM-detected BIRADS3 lesions. The radiologist independently reads DM and 3D
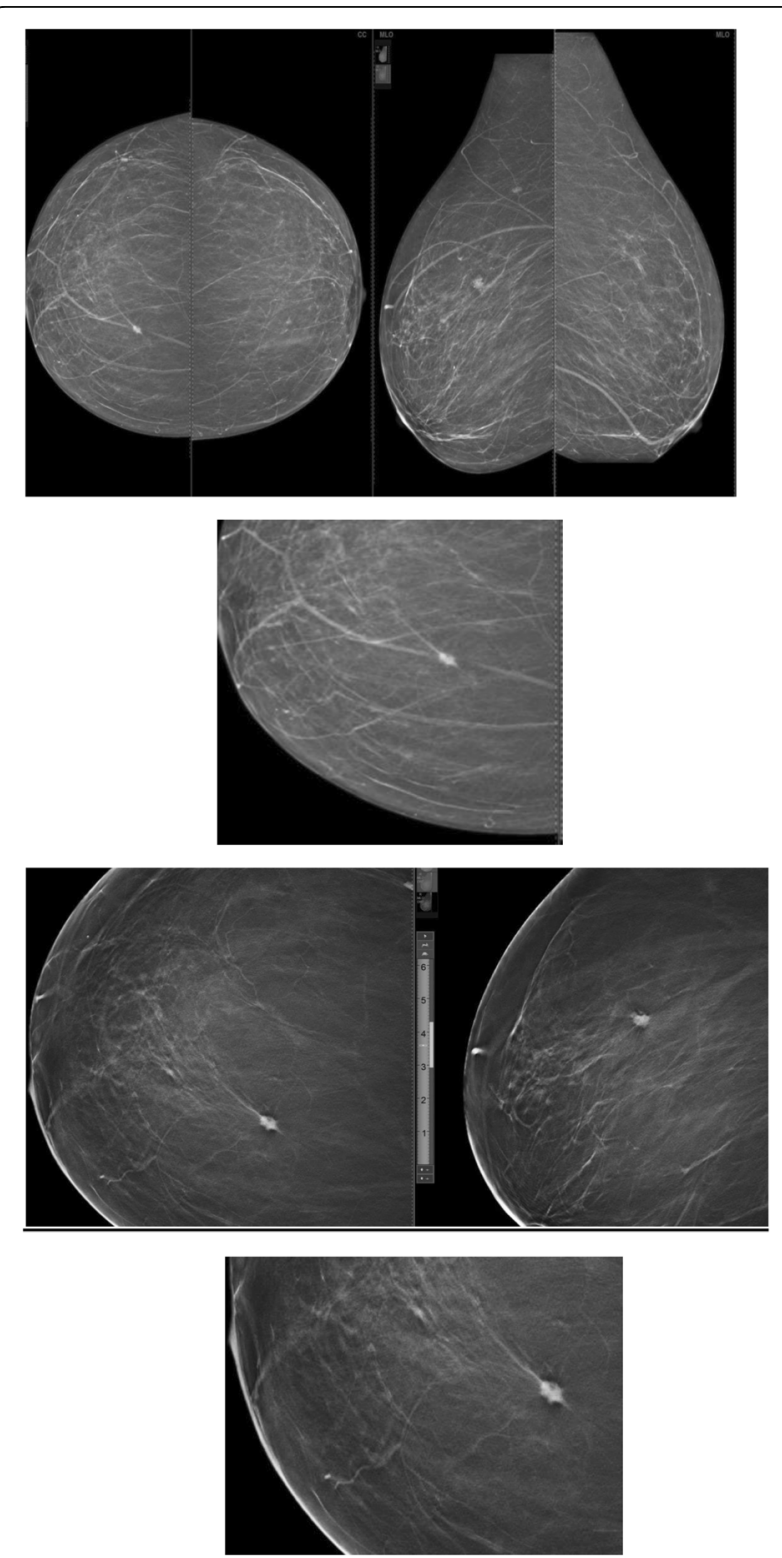

Fig. 7 Sixty-one-year-old female. Mammography CC and MLO views. Bilateral fatty breasts ACR A. Right UIQ small oval ill-defined mass lesion BIRADS 3. Tomosynthesis of the right breast CC and MLO views. The margin of the lesion appeared speculated on 3D digital tomosynthesis images (BIRADS 4). The tomosynthesis has better margin characterization, which easily detected the spiculated margin of this lesion and upgraded the BIRADS category from 3 to 4 ultrasound-confirmed speculated borders 
DBT. Only $22.8 \%$ of BIRADS 3 lesions (82/360) did not change their BIRADS after tomosynthesis, while 77.2\% (278/360) were re-classified according to the BIRADS system. TS "down-staged" BIRADS 3 lesions to BIRADS 1 or 2 categories in most of the cases $(263 / 360)$ and "upstaged" 15 cases to BIRADS 4 and 5 [8].

Helyie subjectively compared 3D DBT when characterizing known masses, architectural distortions, or asymmetries. The study included the mammography of 25 women with known masses. After review of the examinations, radiologists rated their relative preference in terms of classifying the finding in question twice; one time when aided by the additional views and another when aided by 3D DBT. The diagnostic BIRADS rating of both examinations were correlated. They found that DM and 3D DBT (combined) were perceived to be better for diagnosis in $50 \%$ of cases. They concluded that 3D DBT might be an effective alternative to the additional mammographic views in most cases mostly if the presentation of the concerned lesion was not calcification [5].

Skaane verified that DBT added more in defining the shape and margins of breast lesions by decreasing the
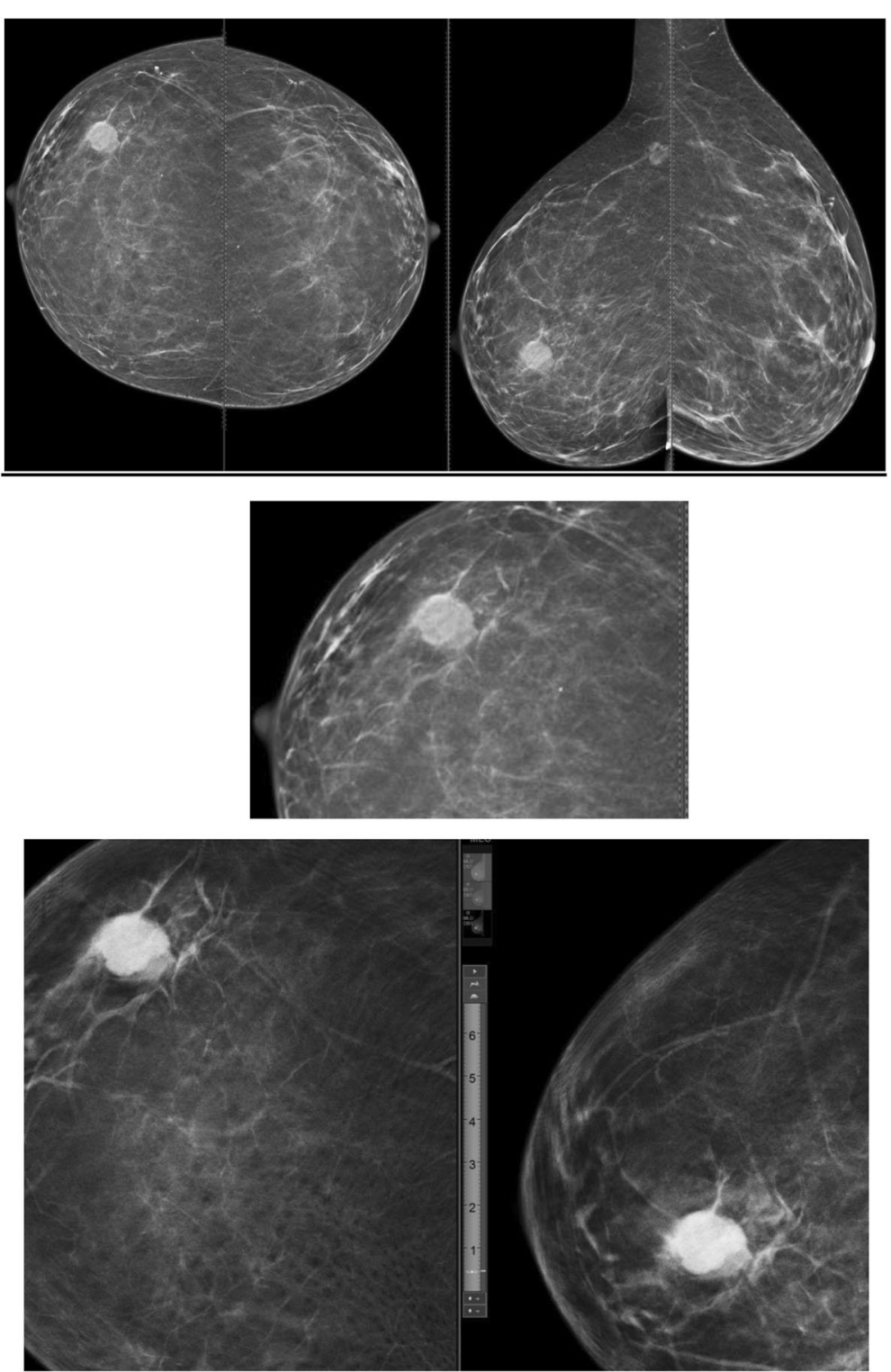

Fig. 8 Fifty-six-year-old female. Mammogram CC and MLO views. Bilateral fatty breasts ACR A. Right LOQ rounded partially well-defined partially obscured dense lesion BIRADS 3. Tomosynthesis of the right breast CC and MLO views. 3D digital breast tomosynthesis showed irregular micro lobulated margin with related architectural distortion of the right LOQ dense mass lesion BIRADS 4. Tomosynthesis detected the fine microlobulations in the breast lesion which was defined as a rather well-defined one by mammography and so shifted the BIRADS from probably benign 3 to suspicious lesion 4 which was pathologically proved to be duct carcinoma 
overlapping tissue, and as a result the ability to differentiate between superimposed tissue and breast lesions would be improved [9].

STORM-2 demonstrated a significant increase in cancer detection rate (CDR) when DBT was added to 2D FFDM (8.5 per 1000 2D+DBT, 6.3 per 1000 2D FFDM). This improvement was maintained and actually slightly increased with s2D+DBT (8.8 per 1000). Incremental CDRs over 2D alone were also similar between s2D+DBT $(+2.5$ per 1000) and $2 \mathrm{D}+\mathrm{DBT}(+2.2$ per 1000$)$. The most significant improvements in cancer detection were seen in women $<60$ years of age and women with dense breasts. As biennial screening is the standard in European screening programs, the CDRs reported in STORM-2 are higher than typical North American practices [10].

Aujero et al.'s results showed a slight increase in cancer detection from 2D FFDM (5.3 per 1000) to 2D+DBT (6.4 per 1000), which was maintained with s2D+DBT (6.1 per 1000). Notably, the percentage of invasive cancers detected with s2D+DBT was significantly higher than 2D and 2D+DBT (s2D+DBT 76.5\%, 2D 61\%, 2D+DBT 61.3\%; $p<0.01$ ), without a loss in in situ cancer detection. This was thought to reflect a learning curve of using DBT, as the s2D+DBT studies were interpreted after some years of experience with DBT [11].

In our study, $60 \%$ of the BIRADS 3 lesions changed their BIRADS by 3 D DBT $48.3 \%$ were down staged to BIRADS 1 and 2, and $11.7 \%$ were "upstaged" to a higher BIRADS. Forty percent of the BIRADS 3 lesions did not change their BIRADS. So 3D DBT significantly reduced the need for additional mammographic views and as well the frequent follow-up studies as it gave better characterization for all BIRADS 3 lesions and reduced the stress levels in women. DBT did not show any false-negative results in our study and it did not miss any cancers.

So the ability to scroll through the three-dimensional data set for a particular view helps in eliminating the overlap of tissues seen in two-dimensional images and better resolution of the internal contents leading to better diagnostic capabilities.

Though the primary role of screening mammography was early detection of breast cancer, tomosynthesis came in with numerous advantages that included the high specificity in benign breast lesions detection and as well as categorization of benign versus malignant lesions. This in turn would reduce the need for additional time-consuming imaging such as special mammographic views or sono-mammography thereby increasing the efficacy of the test by reducing the additional radiation dose, time, and money. At the same time, it reduced the patient's anxiety by avoiding unnecessary recalls.

Therefore, according to our study results, we highly recommend tomosynthesis as a diagnostic algorithm tool in patients with mammography-detected BIRADS 3 lesions (Figs. 4, 5, 6, 7, and 8).

\section{Conclusion}

3D DBT significantly reduced the need for additional mammographic views and frequent follow-up studies as it gave better characterization for all BIRADS 3 lesions. 3D DBT did not show any false-negative results in this study and it did not miss any cancers. In addition, reduction of numerous mammographic controls examination reduced the stress levels in women. Therefore, 3D digital breast tomosynthesis should be applied in the diagnostic algorithm in patients with mammographydetected BIRADS 3 lesions.

\section{Abbreviations \\ BIRADS: Breast imaging-reporting and data system; DBT: Digital breast tomosynthesis; DM: Digital mammography}

\section{Acknowledgements}

First and foremost, thanks to Allah, the most beneficial and most merciful. It is but for His mercy that we can put through in life.

\section{Authors' contributions}

EA and LA participated in the performance of the research, writing, reading, and approving the final manuscript.

\section{Funding}

Not applicable

\section{Availability of data and materials}

The datasets used and analyzed during the current study are available from the corresponding author on reasonable request.

\section{Ethics approval and consent to participate}

The study is a prospective study that was reviewed by the Ethics Committee of Radiology Departments and was approved by the review board that is related to our university. Patients included gave informed written consent to use their data in research work. No applicable reference number.

\section{Consent for publication}

All patients included in this research gave written consent to publish the data contained within this study. If the patient was less than 16 years old, deceased, or unconscious when consent for publication was requested, written informed consent for the publication of this data was given by their parent or legal guardian.

\section{Competing interests}

The authors declare that they have no competing interests.

Received: 13 August 2019 Accepted: 16 September 2019 Published online: 13 November 2019

\section{References}

1. Ferlay J, Soerjomataram I, Ervik M, Dikshit R, Eser S, Mathers C, Rebelo M, Parkin DM, Forman D, Bray F (2014) GLOBOCAN 2012 v1.1, Cancer incidence and mortality worldwide: IARC CancerBase No. 11. International Agency for Research on Cancer, Lyon

2. Teertstra H, Loo C, van den Bosch M et al (2010) Breast tomosynthesis in clinical practice: initial results. EurRadiol 20(1):16-24

3. Mandelson MT, Oestreicher N, Porter PL, White D, Finder CA, Tapllin SH, White E (2000) Breast density as a predictor of mammographic detection: comparison of interval- and screen-detected cancers. J Natl Cancer Inst 92:1081-1087

4. Poplack SP, Tosteson TD, Kogel CA et al (2007) Digital breast tomosynthesis: initial experience in 98 women with abnormal digital screening mammography. AJR 189:616-623

5. Helyie MA (2010) Digital mammography imaging: breast tomosynthesis and advanced applications. Radiol Clin North Am 48(5):917-929 Review

6. Durand MA (2018) Synthesized mammography: clinical evidence, appearance, and implementationby. Diagnostics (Basel) 8(2):22 Department of Radiology, Yale University School of Medicine, New Haven, CT 06412, USA 
7. Yuranga Weerakkody, Radswiki, et al, Breast density, Radiopaedia, 2015

8. Bunovic NP, Prvulovic M, Koprivsek K, Kamenica S, et al: The value of breast tomosynthesis in the assessment of BIRADS 3 lesions. 2014. ECR/C-1905

9. Skaane P (2009) Studies comparing screen-film mammography and full-field digital mammography in breast cancer screening: updated review. Acta Radiol 50(1):3-14

10. Ambinder E, Harvey SC, Panigrahi B, Woods RW (2016) Clinical screening performance of tomosynthesis with synthesized 2D mammograms compared to tomosytheisis with full field digital mammography. Proceedings of the Radiological Society of North America Annual Meeting, Chicago, 27 November-2 December

11. Gilbert FJ, Tucker L, Gillan M, Willsher P, Cooke J, Duncan KA, Michell MJ, Dobson HM, Lim YY, Suaris T et al (2015) Accuracy of digital breast tomosynthesis for depicting breast cancer subgroups in a UK retrospective reading study (TOMMY trial). Radiology 277:697-706

\section{Publisher's Note}

Springer Nature remains neutral with regard to jurisdictional claims in published maps and institutional affiliations.

\section{Submit your manuscript to a SpringerOpen ${ }^{\circ}$ journal and benefit from:}

- Convenient online submission

- Rigorous peer review

- Open access: articles freely available online

- High visibility within the field

Retaining the copyright to your article

Submit your next manuscript at $\boldsymbol{\nabla}$ springeropen.com 